\title{
Development of cooling technologies for SMES
}

\author{
Naoki HIRANO ${ }^{* 1, \dagger}$,Tomonori WATANABE ${ }^{* 1}$ and Shigeo NAGAYA ${ }^{* 1}$
}

\begin{abstract}
A superconducting magnetic energy storage (SMES) system has good characteristics as energy storage equipment in electric power systems such as high efficiency, quick response, no deterioration in repetition operation, and so on. Since 1991, the Agency for National Resources and Energy Japan has carried out a national project to develop an SMES for power control in power systems. Moreover, SMES has been developed to bridge for instantaneous voltage dips since 2003. A field test of 5 MVA SMES for bridging instantaneous voltage dips was carried out on an advanced large liquid crystal TV plant in Kameyama from July 2003. Before that, a 10 MVA SMES system was working there. After the field test, the commercial SMES for instantaneous voltage dips is working there. In 2015, three commercial SMES units for bridging instantaneous voltage dips are operating in Japan. These SMESs are all equipped with metal superconducting coils. When considering cost-reduction, size-reduction, and maintenance of the SMES in the future, it is necessary to develop a SMES with a high-temperature superconductor and improve the efficiency of its cooling system. We have also developed and conducted operation tests of SMES with a Bi2212 oxide superconductor and high performance cryocooler which enable energy conservation operation by inverter control. In this paper, developed results on cooling systems and cryocooler for SMES are presented.
\end{abstract}

Keywords: SMES, Cryocooler, Liquid helium, Inverter control innovation

\section{Introduction}

Superconducting Magnetic Energy Storage (SMES) is a method to store electric power without transforming it into other forms of energy by utilizing the characteristics of superconductivity phenomenon so that the electric resistance becomes zero. This phenomenon is that the electric current keeps flowing without attenuating in the closed circuit when the closed circuit is structured with only the coil made by the superconducting wire. While other power storage methods convert the electric energy into, for example, the chemical energy or the rotation energy, the SMES stores electricity as a form of magnetic energy, and, therefore, the characteristics of the SMES include no loss by energy conversion and high storage efficiency. In addition, the SMES also includes the capability to discharge all stored energy in a very short time (about 1 second) and the capability of the storage part to withstand repeated charging and discharging of several ten thousand or several hundred thousand times without deterioration. ${ }^{1)}$ The practical use of the SMES has already been achieved for the application of bridging instantaneous voltage dips, and is operating in three locations in Japan, including the advanced liquid crystal television plant in Kameyama City, in Mie Prefecture. ${ }^{2)}$ In addition, development progressed on the national project from 1991 to 2012 for the SMES for electric power system control which requires higher technology compared with the SMES for bridging instantaneous voltage dips. Among the development activities, the verification experiment for load fluctuation compensation function and electric power system stabilization function

\footnotetext{
*1 $\quad$ Received February 28, 2014

Superconducting Team, Logistics Group, Electric Power R\&D

Center, Chubu Electric Power Co., Inc.

20-1 Kitasekiyama, Ohdaka-cho, Midori-ku, Nagoya, 459-8522 Japan

Chubu Electric Power Co., Inc. Electric Power R\&D Center, 20-1

Kitasekiyama, Ohdaka-cho, Midori-ku, Nagoya, 459-8522 Japan

Hirano.Naoki@chuden.co.jp
}

was conducted in 2007 by incorporating the SMES system into the actual power system. ${ }^{3)}$ This report presents in detail the technical development trend focusing on the cooling equipment of the SMES. This report also describes the conduction cooling technology by the oxide superconducting coil, the energy saving technology of the small-size cryocooler by using inverters that are being developed, and the cooling technology using liquid hydrogen that has recently drawn attention.

\section{Features of the SMES}

The SMES is equipment to store electric energy as a form of magnetic energy by using the features of superconductivity of zero electric resistance. When the electric current flows in a coil made by the superconducting wire, the attenuation time constant of the electric current flowing in the coil becomes infinity due to no electric resistance. During this time if both ends of the coil are connected, the electric current will keep flowing in this circuit without attenuation and will keep generating the magnetic field produced by the electric current. Based on this phenomenon, electric power is kept stored as a form of magnetic energy. SMES has the following features that other storage methods do not: higher conversion efficiency of electric power, instantaneous responsiveness of the input and the output (for example, discharge of the stored energy in one second) independent control of the active and the reactive power, and the long life of the storage part without deterioration against the repetition of charging and discharging several ten or hundred thousand times.

\section{Development trend of the SMES}

As high current can be handled effectively in the superconducting state, the current density can be enhanced and significant downsizing of the electric power equipment can be achieved. Contributed by this feature, the downsizing merit of the equipment with large capacity is significantly realized for many applications. With the 
additional features of high storage efficiency of the electric power and the excellent durability, the SMES system with a storage capacity of several hundred MWh to several GWh class was studied as one of the applications of large-scale power storage technology including load leveling technology between day and night in 1970s. However, such a large SMES system as described above that aims to replace pumped storage hydropower generation is a large-scale system which has to consider utilization of the underground bedrock to support the electromagnetic force generated by the coil, and has many issues to be solved. Therefore, the SMES system for electric power system stabilization and bridging instantaneous voltage dips has been developed first for the application of activation time in seconds, since it has what the market needs and is technically feasible.

In Europe and America, according to past results, some of the SMES systems of 1 to 3 MJ class in capacity with the metal series superconducting coil manufactured by the American company have been introduced for bridging instantaneous voltage dips. Recently in the U.S.A., development of an SMES system with an ultra-high magnetic field with a capacity of $3.4 \mathrm{MJ}$ (a field of up to $30 \mathrm{~T}$ at $4.2 \mathrm{~K}$ ) utilizing the superconducting wire made of Yttrium series was stated as a three-year plan in 2010, and ABB Ltd. plays the main role with SuperPower Inc. and the Brookhaven National Laboratory to promote this development. Also in France, the SMES is being developed for the pulse power supply of electromagnetic propulsion, and evaluation for coil manufacturing is being implemented with the capacity of $0.8 \mathrm{MJ}$ class utilizing the $\mathrm{Bi}$ series superconducting wire.

In Asia, the SMES has already been developed with the capacity of the MJ class with a coil made of metal series superconducting wire in Korea, China, and India. Fullfledged development of the SMES in Japan has commenced for electric power system control as the national project of the Agency of Natural Resources and Energy Japan in cooperation with industry, government, and academia since 1991. Since the prospects for cost reduction of the superconducting coil looked brighter ${ }^{4}$, the bridging instantaneous voltage dips system (which can implement its function through instantaneous discharge action) has already been practically used. ${ }^{2)}$

\section{Cooling equipment of the SMES}

4.1 Cooling system of the SMES for bridging instantaneous voltage dips

The cooling system of the SMES for bridging instantaneous voltage dips using the metal series superconducting coil has been practically established. It has excellent serviceability and maintainability due to the combination of a small-size refrigerator which matches in size of the items to be cooled (including the coil and electric current lead), and does not require a legal supervisor concerning High Pressure Gas Safety Act. A bird's-eye drawing of the storage part including the cooling equipment is shown in Fig. 1. The cryostat that maintains the superconducting coil at a cryogenic temperature is a container with a vacuum heat insulating structure consisting of the helium container in which the superconducting coil is immersed in liquid helium and the radiation shield layer equipped in the gap between the helium container and the outer vacuum container. The GM refrigerator is also installed in the cryostat to cool the radiation shield and the electric current lead to around $60 \mathrm{~K}$. In addition, the cryostat is designed so that the pressure inside of the helium container can be maintained constant by reliquidation of the gasified helium caused by energization of the coil and the intruded heat from outside with the GMJT refrigerator, and that operation can be continued without any replenishment of the liquid helium during operation once it is filled.

\subsection{Cooling system of the SMES for electric power system control}

The equipment manufactured for the field test of the SMES for electric power system control employed the recondensation mechanism (which was not employed in the field test equipment for bridging instantaneous voltage dips), to remove the large amount of heat regularly generated by continuous repetitive operation of the charging and discharging of the SMES. The schematic cooling system of the SMES' field test equipment for electric power system control is shown in Fig. 3. Helium gas at $6.2 \mathrm{~K}$ and $0.6 \mathrm{MPa}$ is fed into the valve box in the cryostat from the heavy duty refrigerator, and is expanded through the JT valve resulting in generation of a gasliquid two-phase flow. This gas-liquid two-phase flow

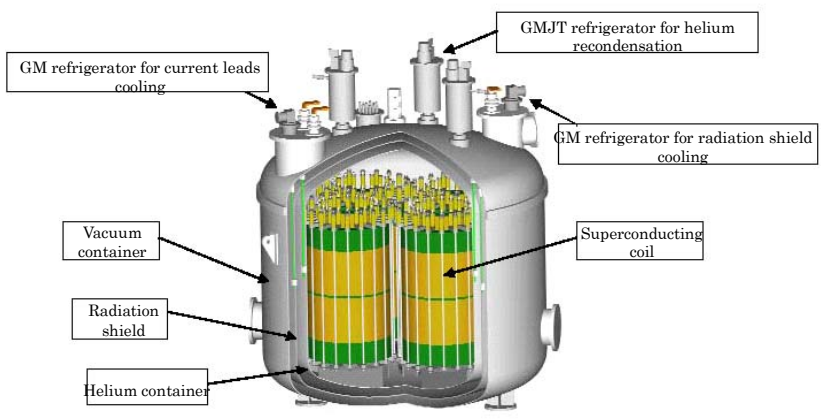

Fig. 1 Bird's-eye drawing of the storage part of the SMES for instantaneous voltage dips compensation

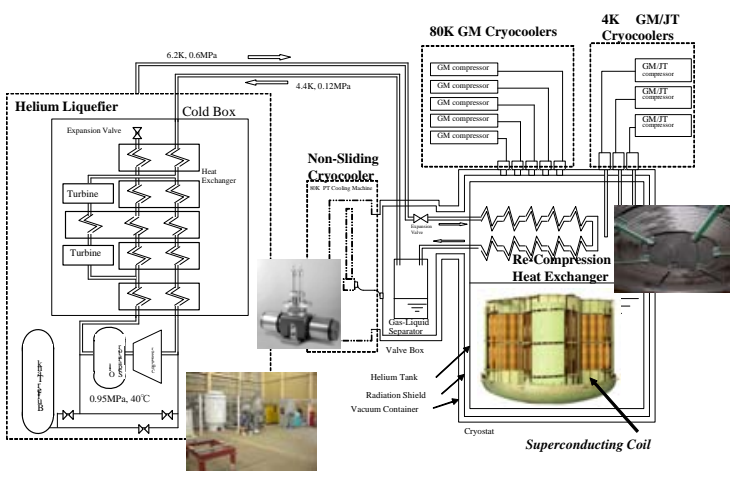

Fig. 2 Schematic diagram of the SMES cooling system for electric power system control with some photographs. 
passes through the re-condensation heat exchanger, attaches to the top part of the cryostat to re-condense the helium gas evaporated by the heat generated by the coil. AS shown in fig.2, the re-condensation heat exchanger is made of the stainless pipe that is wound in a pancake-like shape. Since the amount of heat generated in the superconducting coil is lower than that of the design value, re-condensation operation was not implemented by the heavy duty refrigerator, and the repetitive charging and discharging test was implemented by a recondensation operation of the GMJT cryocooler like the one used in the SMES for bridging instantaneous voltage dips.

4.3 Cooling technology of the oxide series superconducting coil of the SMES

It is confirmed that the superconducting coil has significantly higher thermostability during cooling at a temperature range of $20 \mathrm{~K}$ to $40 \mathrm{~K}$, where the characteristics of the yttrium series superconducting coil are mostly utilized, compared with cooling at the conventional temperature range by liquid helium. Utilization of this high heat capacity enables the SMES to be operated in overload for a short period and to be cooled by the highly efficient refrigerator. However, in the temperature range described above (unlike the temperature range of $4 \mathrm{~K}$ ), it is difficult to achieve a sufficient heat transfer with only the cooling system by solid heat conduction. Therefore, the cooling system promoting heat transfer was developed by circulation of helium gas as shown in Fig. 3. Evaluation of the coil characteristics was performed with conduction cooling by connecting the model coil of the SMES to be evaluated to this cooling system. The model coil is a single pancakelike shape that is made by winding the $\mathrm{Y}$ series superconducting wire and is overlapped in two layers after the insulation process, and the aluminum heat exchanger plates where the cooling tubes are embedded are attached on both surfaces of the two layers. It is verified that the whole coil is cooled below $20 \mathrm{~K}$ by circulation of the helium gas cooled below $20 \mathrm{~K}$ with the light-duty refrigerator. In addition, the coil charge/discharge characteristics were verified by applying a current variation of $400 \mathrm{~A}$ in 10 second cycles to the coil at least 100 times while the model coil was cooled to 20 $\mathrm{K}$. The power supply for charge/discharge test is also shown in Fig.3. We show the typical test result in Fig.4. Temperature stability was confirmed since there was no increase in temperature due to charging and discharging under these operation conditions. ${ }^{5)}$

4.4 Development of the light-duty refrigerator with inverter control

The refrigeration load varies significantly depending on the operation period or stand-by period in the SMES system. However, the conventional refrigerator cannot change its refrigeration capacity. Therefore, the system always has to be operated at the maximum load, and consumes a lot of electric power, requiring reduction. Making the operating frequency of the compressor and the refrigerator variable by inverter control enables the refrigeration system to vary the refrigeration capacity depending on the load. The refrigerator has been developed with a variable capacity and high reliability at $4 \mathrm{~K}, 20 \mathrm{~K}$, and $77 \mathrm{~K}$ to reduce power consumption. Taking an example from the $77 \mathrm{~K}$ inverter refrigerator, the inverter motor is used for the refrigerator driving motor and can be driven with an operation frequency of up to 80 Hz. In addition, the compressor also uses the inverter driven compressor, and can be driven in the frequency range of $30 \mathrm{~Hz}$ to $73 \mathrm{~Hz}$. The measurement results of the refrigeration capacity are shown in Fig. 5 when the

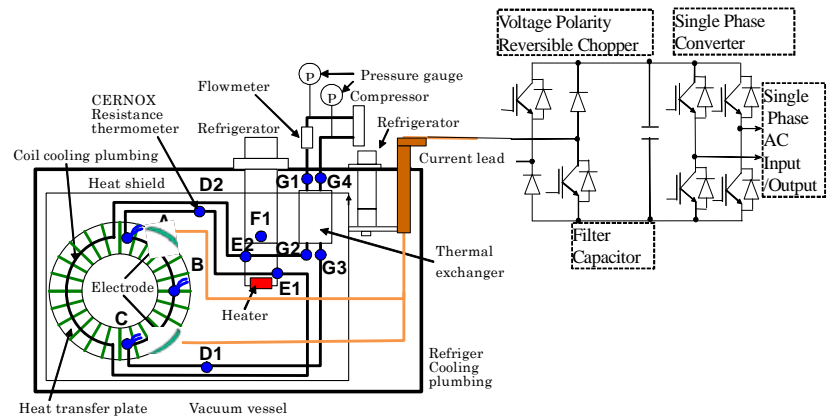

Fig. 3 Helium gas circulation cooling system for charge and discharge Test

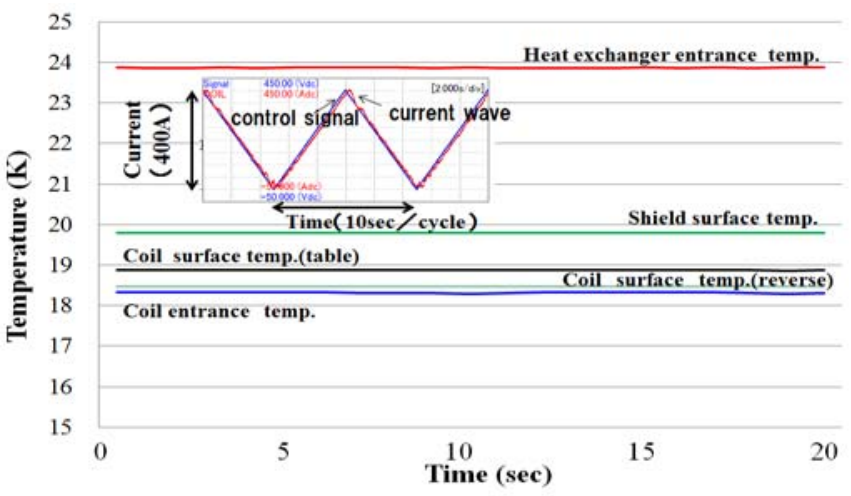

Fig. 4 Typical charge/discharge test result for the model coil cooled by circulation of helium gas.

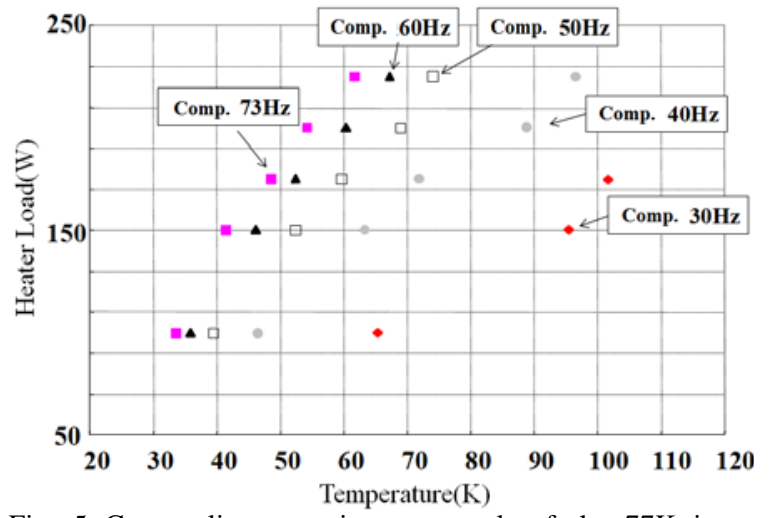

Fig. 5 Cryocooling capacity test result of the $77 \mathrm{~K}$ inverter controlled cryocooler 
driving frequency of the refrigerator is $60 \mathrm{~Hz}$ and one of the compressor is varied. We succeed that the driving performance of the refrigerator was controlled widely by changing the frequency of its compressor. We show cooling temperature depending on frequency of the compressor when we input heater load of $150 \mathrm{~W}$ in Fig.6. If we want to get temperature of $70 \mathrm{~K}$, the frequency of the compressor is $60 \%$ of rated frequency $(60 \mathrm{~Hz})$. Since the consumption electricity is proportional to the frequency of the compressor, we can operate the system by energy saving. The responsiveness of the refrigerator is shown in comparison developed refrigerator with conventional refrigerator in fig 7 . Though the developed inverter refrigerator is decrease its frequency, it is confirm that its responsiveness is the almost same as conventional refrigerator. It is verified that power consumption during the stand-by period can be reduced compared to the conventional equipment, since the operation can be controlled according to the target temperature.

\subsection{The SMES with liquid hydrogen cooling}

A hybrid system is being studied where the renewable energy fluctuations of power generation are separated into long-term and short-term components and the fluctuations in short-term are compensated by the SMES. The characteristics of this system are to store the surplus energy produced by the long-term fluctuations through the means of liquid hydrogen and are to use this liquid hydrogen as refrigerant to reduce the cooling penalty of the SMES. This system allows hydrogen to be produced by the electrolysis of water in case of surplus of electric power, and the electric power is generated by the hydrogen using the fuel cells in case the electric power is required. The model coil was successfully cooled with indirect cooling by thermo-siphon method, and was verified to have current-passing capacity of 200A with a coil made of Bi2212. ${ }^{6}$ Research and development is scheduled in the future on a coil using $\mathrm{MgB}_{2}$ which can be cooled to the temperature of the liquid hydrogen.

\section{Summary}

The cooling equipment for SMES were described mainly in this paper. We have developed the cooling technique for SMES, that is, we can keep the cooling SMES cooled by liquid helium without liquid supplementing using the small refrigerators. The refrigerators has been also developed with a variable cooling capacity and high reliability using inverter control technique to reduce power consumption. Recently, we have started to develop a coil using $\mathrm{MgB}_{2}$ which can be cooled by the liquid hydrogen. It is important to promote the technological development of cost reduction of the SMES and verification for high reliability required by the electric power equipment. Especially, the cryogenic refrigerator and the cooling system which affect the reliability of the superconducting electric power equipment are very important, and continuous development and promotion are sincerely desired.

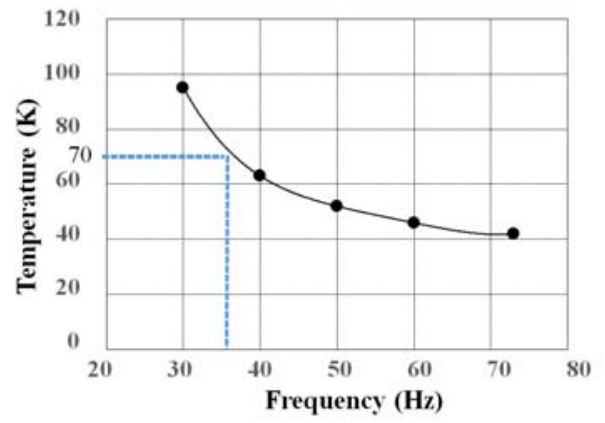

Fig. 6 Cooling temperature depending on frequency of the compressor when we input heater load of $150 \mathrm{~W}$

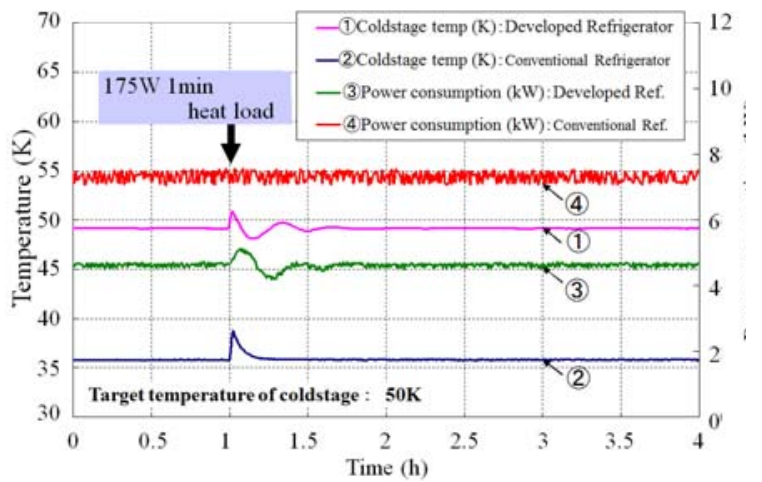

Fig. 7 The responsiveness of the refrigerator comparison with developed refrigerator and conventional refrigerator

Acknowledgements

The part of these present studies was supported by the New Energy and Industrial Technology Development Organization (NEDO) and is sponsored through the ALCA Program by the Japan Science and Technology Agency (JST).

\section{References}

1) S. Nagaya, et al., "Field Test Results of the 5MVA5MJ SMES for Bridging instantaneous Voltage Dips", IEEE Trans. Appl. Supercond. Vol.16, no. 2, 632 (2006).

2) S. Nagaya, et al., "The State of the Art of the Development of SMES for bridging instantaneous voltage dips in Japan”, Cryogenics, 52, 708 (2012).

3) T. Katagiri, et al., "Field test result of 10MVA/20MJ SMES for load fluctuation compensation", IEEE Trans. Appl. Supercond. Vol.19, no. 3, 1993 (2009).

4) Y. Tatsuta, et.al. Cost Reduction Measures for SMES, TEION KOGAKU 40, 5, (2005)

5) S Nagaya, et.al., "Development of a High-Efficiency Conduction Cooling Technology for SMES Coils", Appl. Supercond., Vol. 23, 3, Part: 2 Digital Object Identifier: 10.1109/TASC.2012.2235751 (2013), Article\#: 5602804

6) Y. Makida et al., Performance of a $10 \mathrm{~kJ}$ SMES Model Cooled by Liquid Hydrogen Thermo-Siphon Flow for ASPCS Study, to be published in the proceedings of CEC2015. 